\title{
Enumerating autoreactive $T$ cells in peripheral blood: a big step in diabetes prediction
}

\author{
George S. Eisenbarth ${ }^{1}$ and Brian L. Kotzin ${ }^{2}$ \\ ${ }^{1}$ Departments of Pediatrics, Medicine, and Immunology, Barbara Davis Diabetes Center for Childhood Diabetes and University of Colorado \\ Health Sciences Center, Denver, Colorado, USA \\ ${ }^{2}$ Departments of Medicine and Immunology, University of Colorado Health Sciences Center, Denver, Colorado, USA \\ J. Clin. Invest. 111:179-181 (2003). doi:10.1172/JCI200317621.
}

In this issue of the JCI, Trudeau and colleagues make a bold statement: "We show, we believe for the first time, that the development of an autoimmune disease can be predicted based on the presence of antigen-specific cytotoxic T cells in the peripheral blood." (1). We suspect that, like most "firsts," this one will be challenged. Nevertheless, based on recent workshops showing the difficulty analyzing autoreactive $\mathrm{T}$ cells in patients with type 1 diabetes and in animal models of this disease $(2,3)$, these commentators are inclined to accept this very important "first." Though type 1 diabetes is marked by pancreatic islet $\beta$ cell-specific autoantibodies and can be predicted with highly specific and sensitive autoantibody assays $(4,5), \beta$ cell damage and destruction are mediated by $\mathrm{T}$ cells (both $\mathrm{CD}^{+}$and $\left.\mathrm{CD}^{+}\right)(5,6)$. Thus, quantifying islet-reactive $T$ cells in patients genetically at high risk to develop disease and in patients with prediabetes may be a more direct (and at least complementary) approach to detect $\beta$ cell autoimmunity and predict which patients will go on to develop disease. In type 1 diabetes, assays must be able to identify autoreactive cells in the peripheral blood, not just the target organ and surrounding

\footnotetext{
Address correspondence to: George S. Eisenbarth, Barbara Davis Center for Childhood Diabetes, Box B140, 4200 East Ninth Avenue, Denver, Colorado 80262, USA Phone: (303) 315-4891; Fax: (303) 315-4892; E-mail: george.eisenbarth@uchsc.edu. Conflict of interest: The authors have declared that no conflict of interest exists. Nonstandard abbreviations used: $T$ cell receptor (TCR); glutamic acid decarboxylase (GAD).
}

lymphoid tissue where they accumulate, since the blood is the only place where sampling can be routinely done. However, the hurdles have been so high and progress so limited, it has been debated whether autoreactive $T$ cells circulate in sufficient quantities to be directly detected. Assays that quantify and characterize the function of islet-specific $\mathrm{T}$ cells in blood are also needed to monitor therapeutic trials in type 1 diabetes. To date, two very large trials of therapies to prevent type 1 diabetes have failed to show an effect on an apparently inexorable disease progression (7). Although one of the therapies was directed at insulin-specific T cells, no reliable assays monitored effects on this cell population. The efforts have been compared to testing therapies that prevent hypertensive kidney disease without being able to measure blood pressure.

Trudeau and colleagues (1) report that, using an MHC class I tetramer with a mimotope peptide and flow cytometry, they can directly count the number of autoreactive $\mathrm{T}$ lymphocytes. Increased numbers of such $T$ cells in peripheral blood reliably distinguish NOD mice that do progress to diabetes from those that do not. For the nonimmunologist, MHC-peptide tetramers (or multimers) are soluble complexes of MHC molecules (HLA in humans or $\mathrm{H}-2$ in mice) with the same peptide bound in the MHC groove (8, 9). T cells, through their surface $T$ cell receptors (TCRs), recognize the MHCpeptide complex on the surface of antigen-presenting cells. Compared to antibodies, TCRs generally have a much lower affinity for their antigen (MHC-peptide). To increase avidity for the TCR on antigen-specific T cells, the MHC-peptide complex is engineered to be a multimer. This is accomplished by attaching biotin to the MHC component and then binding multiple MHC-peptide complexes to streptavidin through its four biotin-binding sites (Figure 1). Thus, although a single MHC-peptide complex has too low affinity to stay bound to a TCR, multimers (via cooperative multivalent binding) have much higher avidity and form stable interactions. The streptavidin is labeled with a fluorescent marker, and binding of the tetramer to $\mathrm{T}$ cells can be detected with a flow cytometer. Unlike other assay techniques that require in vitro $\mathrm{T}$ cell proliferation or cytokine production, fluorescently-labeled MHC-peptide tetramers permit direct detection and isolation of antigen-specific $\mathrm{T}$ cells, independent of $\mathrm{T}$ cell function.

A prodigious amount of earlier research on $\mathrm{T}$ cells in NOD mice provides the foundation for the current study. The actual endogenous peptides recognized by islet-infiltrating $\mathrm{CD}^{+} \mathrm{T}$ cells are mostly unknown, so random peptide libraries were screened and a peptide "mimotope" designated NRP (KYNKANWFL), recognized by a distinct subset of the autoreactive clones, was identified (10). T cells with this reactivity were shown to predominate in cultured islets of NOD mice just before the onset of diabetes (11). Furthermore, TCR transgenic NOD mice that expressed this NRP-reactive TCR had accelerated diabetes, providing additional evidence for a role of this $\mathrm{T}$ cell reactivity in disease. The mimotope peptide was modified to a peptide 

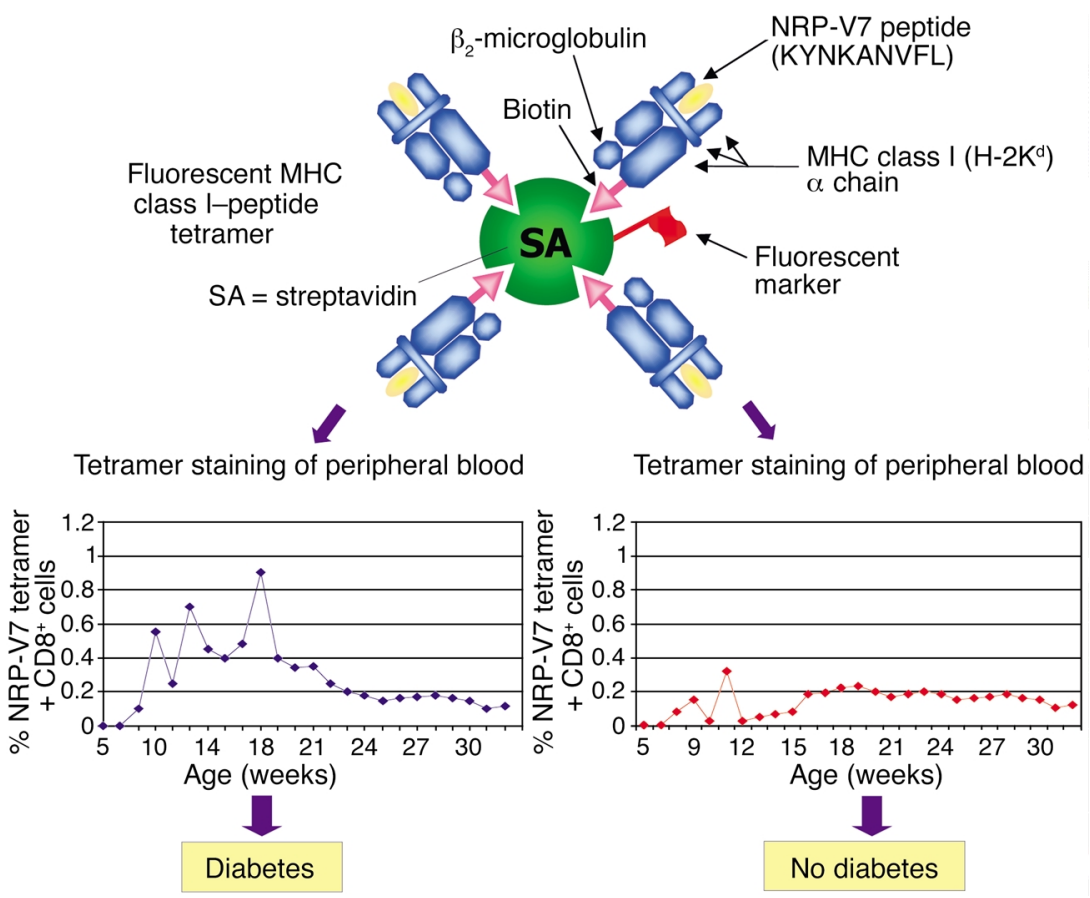

Figure 1

Schematic of fluorescent MHC class I-peptide tetramer used in the study by Trudeau and colleagues ( 1 ) to enumerate autoreactive $C D 8^{+} T$ cells in the peripheral blood of NOD mice. The percentages of $\mathrm{CD}^{+}$cells specific for $\mathrm{H}^{-K^{d}} / \mathrm{NRP}-\mathrm{V} 7$ peptide in multiple peripheral blood samples taken from each mouse were determined by flow cytometry. The patterns for mice that went on to develop diabetes (left) and mice that failed to develop diabetes by 32 weeks of age (right) are shown in the lower histograms.

that, when complexed with $\mathrm{H} 2-\mathrm{K}^{\mathrm{d}}$ (the presenting MHC class I molecules in the NOD mouse), had a higher avidity for the islet-reactive TCR $(11,12)$. A tetramer with the modified mimotope NRP-V7 (valine at position 7) was better at detecting $T$ cells in the infiltrated islets, and only this tetramer could identify $\mathrm{T}$ cells in the peripheral blood (1). Tetramers with the NRP peptides are not the first MHC-peptide class I tetramers shown to react with isletinfiltrating cells in NOD mice. For example, Wong and coworkers (13) described an $\mathrm{H} 2-\mathrm{K}^{\mathrm{d}}$-insulin peptide tetramer that bound to a high percentage of islet-infiltrating cells. Although $\mathrm{CD}^{+} \mathrm{T}$ cells specific for the insulin peptide may be important early pathogenic $T$ cells in the diabetogenic process, these cells never expanded to a high percentage of islet-reactive cells and were not detected in the peripheral blood in the current study.

The success of the current study may suggest certain general principles for others interested in identifying antigen-specific $\mathrm{T}$ cells in the blood of patients with autoimmune diseases. One may be that MHC class I tetramers will turn out to be much more useful for studying circulating $\mathrm{T}$ cells than MHC class II-peptide tetramers, which stain antigen-specific $\mathrm{CD} 4^{+}$cells. Studies have suggested that the frequency of circulating antigen-specific $\mathrm{CD}^{+}$cells is much lower than $\mathrm{CD}^{+}$cells, even in the same disease process $(8,9)$. In most studies of humans for peptide-specific $\mathrm{CD}^{+} \mathrm{T}$ cells, tetramer-positive cells have not been detected in freshly isolated peripheral blood, and in nearly every case, in vitro expansion of antigenreactive cells has been required to document the existence of circulating antigen-specific $\mathrm{CD} 4^{+} \mathrm{T}$ cells in type 1 diabetes. MHC class II tetramers are being used in the human disease to detect glutamic acid decarboxylasereactive (GAD-reactive) T cells (14). In patients with recent-onset disease, expansion in culture was necessary to estimate that only one in $30,000 \mathrm{CD} 4^{+}$ $\mathrm{T}$ cells in freshly isolated blood is tetramer reactive. This frequency is well below the limit of detection for direct tetramer binding by flow cytometry. Although MHC class II tetramers with mimotope peptides have been described for a particular $\mathrm{CD} 4^{+} \mathrm{T}$ cell clone (BDC2.5) in the NOD model, it is not clear that the number of these $\mathrm{CD} 4^{+} \mathrm{T}$ cells is increased in prediabetic mice (15). A focus on islet-specific $\mathrm{CD}^{+} \mathrm{T}$ cells in NOD mice (and in human type 1 diabetes) is also supported by considerable evidence showing that this population is a major contributor to tissue damage in the animal model (16).

The sensitivity of detection with MHC-peptide tetramers is proportional to the intensity of fluorescent staining of the $\mathrm{T}$ cell. Perhaps the most important variable in this process is the affinity of the MHCpeptide complex for the TCR on the antigen-specific $T$ cell (other variables include the number of TCRs on the surface and the functional state of the $\mathrm{T}$ cell) $(17,18)$. Therefore, another critical aspect of the current study is that success was attained by engineering a mimic of the NRP epitope (replacing a valine for a tryptophan at P7 in the peptide), which resulted in an MHC-peptide complex that had higher affinity for the TCR of the autoreactive cell $(11,12)$. Without knowing the endogenous antigen that underlies NRP reactivity, we cannot know whether this modified peptide more closely resembles the true selfantigen. However, there are a number of studies to suggest that autoreactive $\mathrm{T}$ cells, including those in disease, may be relatively low avidity compared to $\mathrm{T}$ cells elicited by foreign antigens. Thus, the highest avidity self-reactive cells are deleted through intrathymic and peripheral immune tolerance mechanisms, leaving cells with lower avidity around to be activated during the generation of autoimmunity. The higher avidity of $\mathrm{T}$ cells specific for foreign antigens may explain the greater usefulness of MHC-peptide tetramers in infectious diseases compared to autoimmune diseases (when the autoantigen is known) (8).

The success of the current study also allows for some interesting insight into the pathogenic process. For example, the tetramer-staining cells in blood seemed to appear in cycles, prior to the onset of hyperglycemia (Figure 1). The authors speculate that these cycles reflect waves of clonal proliferation near the target organ, possibly leading sequentially to $T$ cell clones with higher avidity for self antigen (11). 
Perhaps these waves are tied to the release of antigen from $\beta$ cell death and activation of NRP-reactive $\mathrm{CD}^{+} \mathrm{T}$ cells in the pancreatic lymph nodes (16). Related to this finding is the observation that detection of elevated percentages of autoreactive $T$ cells was facilitated by repeated measurements of blood specimens. Even in mice destined to develop hyperglycemia, most samples contained low (undetectable) levels of autoreactive cells (Figure 1). Finally, the number of tetramer-binding $\mathrm{CD}^{+}$ cells progressively decreased in the blood after the development of diabetes, perhaps reflecting the progressive loss of $\beta$ cell antigen as these cells were destroyed. This finding implies that studies of prediabetics may be more revealing than those of patients with long-term diabetes.

The report by Trudeau et al. (1) immediately raises some critical questions that are already probably in the process of investigation. For example, the actual self-antigen ( $\beta$ cell-antigen) recognized by the $\mathrm{CD} 8^{+}$NRP-reactive $\mathrm{T}$ cell has not been reported. Once described, it will then be clear whether the modification of peptide sequence improved affinity artificially or resulted in a better match to the endogenous antigen. The relative avidity of the autoreactive NRP-reactive clones for the native peptide will also be clear.

Previous studies have shown that injections with a higher-affinity mimic of the NRP peptide prevents diabetes in NOD mice and are associated with decreased $\beta$ cell specific cytotoxicity (11). It will be of interest to determine whether peptide-mediated effects on function or shifts in the population of NRP-reactive cells can be detected in the peripheral blood by the techniques described in the current report. Though there is increasing evidence that insulin is a key islet autoantigen and that disease suppression and induction can be mediated with insulin peptide B:9-23 (19), no MHC class II tetramer is currently available for analysis of insulin-reactive T cells. Depending on the role of the NRP molecule in cell development, eventually it may also be possible to knock out or alter the NRP molecule to determine if it is a critical target for $\beta$ cell destruction. Mice have two different insulin gene loci, and preliminary studies of mice with selective knockouts of each of these genes further support the hypothesis that insulin is a critical autoantigen in the disease process.

There is continuing debate as to whether the actual development of diabetes is dependent upon a change in the immune system just prior to overt hyperglycemia. This remains a formal possibility, but studies of avidity maturation of NRP-reactive T cells (11) and ability to predict diabetes suggest a more progressive model of disease development. Characterization of the circulating and islet-infiltrating NRP-reactive $T$ cells is now possible, and TCR avidity can be analyzed over time.

In addition to the considerable evidence that $\mathrm{CD}^{+} \mathrm{T}$ cells are centrally involved in the $\beta$ cell damage in NOD mice (16), there is some evidence that HLA class I alleles may influence the development of disease. For instance, the HLA-A2 allele, when introduced as a transgene into NOD mice, accelerates the development of diabetes (20). Furthermore, individuals with HLA-A1 and -A2 haplotypes may be at increased risk to develop disease. The current report should stimulate a major effort to identify autoreactive HLA class I-restricted $\mathrm{T}$ cells in humans so that these elegant studies can be extended to prediabetic patients, whose families are anxiously awaiting therapies as effective in disease prevention as the many we have for the NOD mouse.

1. Trudeau, J.D., et al. 2003. Prediction of spontaneous autoimmune diabetes in NOD mice by quantification of autoreactive $\mathrm{T}$ cells in peripheral blood. J. Clin. Invest. 111:217-223. doi:10.1172/JCI200316409.

2. Kaufman, D.L., et al. 2001. Report from the 1st International NOD Mouse T-Cell Workshop and the follow-up mini-workshop. Diabetes. 50:2459-2463.
3. Roep, B.O., et al. 1999. Autoreactive T cell responses in insulin-dependent (type 1) diabetes mellitus. Report of the First International Workshop for Standardization of $\mathrm{T}$ Cell Assays. J. Autoimmun. 13:267-282.

4. Yu, L., et al. 2000. Early expression of antiinsulin autoantibodies in humans and the NOD mouse: evidence for early determination of subsequent diabetes. Proc. Natl. Acad. Sci. USA. 97:1701-1706.

5. Notkins, A.L., and Lernmark, A. 2001. Autoimmune type 1 diabetes: resolved and unresolved issues. J. Clin. Invest. 108:1247-1252. doi:10.1172/JCI200114257.

6. Tisch, R., and McDevitt, H. 1996. Insulin-dependent diabetes mellitus. Cell. 85:291-297.

7. Diabetes Prevention Trial Study Group. 2002 Effects of insulin in relatives of patients with type 1 diabetes mellitus. N. Engl. J. Med. 346:1685-1691.

8. Klenerman, P., Cerundolo, V., and Dunbar, P.R 2002. Tracking T cells with tetramers: new tales from new tools. Nat. Rev. Immunol. 2:263-272.

9. Nepom, G.T., et al. 2002. HLA class II tetramers: tools for direct analysis of antigen-specific CD4+ T cells. Arthritis Rheum. 46:5-12.

10. Anderson, B., Park, B.-J., Verdaguer, J., Amrani, A and Santamaria, P. 1999. Prevalent CD8+ T cell response against one peptide/MHC complex in autoimmune diabetes. Proc. Natl. Acad. Sci. USA. 96:9311-9316.

11. Amrani, A., et al. 2000. Progression of autoimmune diabetes driven by avidity maturation of a T-cell population. Nature. 406:739-742.

12. Amrani, A., et al. 2001. Expansion of the antigenic repertoire of a single $\mathrm{T}$ cell receptor upon $\mathrm{T}$ cell activation. J. Immunol. 167:655-666.

13. Wong, F.S., et al. 1999. Identification of an MHC class I-restricted autoantigen in type 1 diabetes by screening an organ-specific cDNA library. Nat. Med. 5:1026-1031.

14. Reijonen, H., et al. 2002. Detection of GAD65specific T-cells by major histocompatibility complex class II tetramers in type 1 diabetic patients and at-risk subjects. Diabetes. 51:1375-1382.

15. Dobbs, C., and Haskins, K. 2001. Comparison of a $\mathrm{T}$ cell clone and of T cells from a TCR transgenic mouse: TCR transgenic T cells specific for self-antigen are atypical. J. Immunol. 166:2495-2504

16. Liblau, R.S., Wong, F.S., Mars, L.T., and Santamaria, P. 2002. Autoreactive CD8 T cells in organspecific autoimmunity: emerging targets for therapeutic intervention. Immunity. 17:1-6.

17. Crawford, F., Kozono, H., White, J., Marrack, P. and Kappler, J. 1998. Detection of antigen-specific T cells with multivalent soluble class II MHC covalent peptide complexes. Immunity. 8:675-682.

18. Fahmy, T.M., Bieler, J.G., Edidin, M., and Schneck, J.P. 2001. Increased TCR avidity after T cell activation: a mechanism for sensing low-density antigen. Immunity. 14:135-143.

19. Moriyama, H., et al. 2002. Induction and acceleration of insulitis/diabetes in mice with a viral mimic (polyinosinic-polycytidylic acid) and an insulin self-peptide. Proc. Natl. Acad. Sci. USA. 99:5539-5544.

20. Marron, M.P., Graser, R.T., Chapman, H.D., and Serreze, D.V. 2002. Functional evidence for the mediation of diabetogenic $\mathrm{T}$ cell responses by HLA-A2.1 MHC class I molecules through transgenic expression in NOD mice. Proc. Natl. Acad. Sci. USA. 99:13753-13758. 